\title{
Gestión centralizada de camas de Unidad de Cuidado Intensivo e Intermedio por el Centro Regulador de Urgencias y Emergencias de Bogotá ante el COVID-19*
}

\section{Centralized management of Intensive and Intermediate Care Unit beds by the Emergency Care Regulation Center of Bogotá facing the COVID-19}

\section{Gestão centralizada de leitos de Unidade de Terapia Intensiva e Semi-intensiva pelo Centro Regulador de Urgências e Emergências de Bogotá perante a COVID-19}

Recibido: 30 de Noviembre de 2020. Aceptado: 25 de Febrero de 2021. Publicado: 30 de diciembre de 2021.

DOI: https://doi.org/10.11144/Javeriana.rgps20.gccu

\author{
Andrés José Álvarez Villegas ${ }^{a}$ \\ Secretaría Distrital de Salud de Bogotá, Colombia \\ ORCID: https://orcid.org/0000-0002-1814-9933 \\ Ángela María Roa Saldaña \\ Secretaría Distrital de Salud de Bogotá, Colombia \\ ORCID: https://orcid.org/0000-0002-8919-9487 \\ Edizabeth Ramírez Rodríguez \\ Secretaría Distrital de Salud de Bogotá, Colombia \\ ORCID: https://orcid.org/0000-0001-8662-820X
}

\begin{abstract}
Para citar este artículo Álvarez AJ, Roa AM, Ramírez E. Gestión centralizada de camas de Unidad de Cuidado Intensivo e Intermedio por el Centro Regulador de Urgencias y Emergencias de Bogotá ante el COVID-19. Rev Gerenc Polit Salud. 2021;20. https://doi.org/10.11144/Javeriana.rgps20.gccu
\end{abstract}

* Artículo de investigación

a Autor de correspondencia. Correo electrónico: ajalvarez@saludcapital.gov.co 


\section{Resumen}

Introducción. En el marco de la emergencia sanitaria se ha presentado un aumento de casos críticos de COVID-19 en las Unidades de Cuidados Intensivos (UCI) e Intermedios (UCIM), lo que implica desarrollar estrategias de coordinación. Objetivo. Describir la estrategia implementada en Bogotá para la coordinación de camas de las UCI y UCIM, frente el aumento de casos críticos de COVID-19. Metodología. Se realizó un estudio descriptivo con los datos de los tableros de control de la Dirección de Urgencias y los indicadores de SaluData. Se contrastaron los puntos esenciales de la estrategia distrital y el porcentaje de ocupación en los servicios UCI y UCIM con corte cada 12 horas. Resultados. Se describe el diseño y la implementación de la estrategia; los indicadores de porcentaje de ocupación específicos para camas UCI, UCIM; los indicadores de hospitalización para casos COVID-19 y no COVID-19; y los parámetros de alta demanda en un rango mayor o igual a 50 en el porcentaje de ocupación de camas UCI adulto, por un periodo continuo mayor a 24 horas. Se determinaron los niveles de alerta teniendo en cuenta dichos porcentajes. Igualmente, fueron tomados en cuenta los procedimientos, instrumentos y canales de comunicación necesarios para la regulación de la oferta de camas. Conclusión. La gestión centralizada de camas UCI por parte del Centro Regulador ha favorecido la prestación de servicios de salud a pacientes en estado crítico.

Palabras clave: Infecciones por Coronavirus, unidades de cuidados intensivos, servicios de salud, ocupación de camas, reconversión de camas.

\section{Abstract}

Introduction. In the context of the health emergency, there has been an increase in the number of critical cases of COVID-19 in the Intensive and Intermediate Care Units (ICU, IMCU), indicating the need to develop strategies for coordination. Objective. To describe the approach implemented in Bogota for the bed occupancy coordination on the ICU and IMCU, facing an increase in critical COVID-19 cases. Methods. We conducted a descriptive study using Emergency Division databoards and SaluData indicators. Additionally, we made a comparison between the essential points of the Capital's strategy and the bed occupancy rate in ICU and IMCU services at 12-hour intervals. Results. We described the design and implementation of the strategy, the bed occupancy rate indicators for COVID-19 cases, the hospitalization indicators for COVID-19 and non-COVID-19 cases, and the parameters of high demand (adult ICU beds occupancy percentage $50 \%$ or highest) for more than 24 hours. Taking into account these percentages we determined the alerts levels. Procedures, instruments, and channels of communication necessaries for supplying beds were considered as well. Conclusion. Centralized management of ICU beds by the Regulation Center has been favorable for the health services provided to patients in a critical state.

Keywords: Coronavirus infections, intensive care units, health services, bed occupancy, bed reconversion.

\section{Resumo}

Introdução. No marco da emergência sanitária, tem se presentado um aumento de casos críticos de COVID-19 nas Unidades de Cuidados Intensivos (UCI, UCIM), o que implica desenvolver estratégias de coordenação. Objetivo. Descrever a estratégia implementada em Bogotá para a coordenação de camas de UCI e UCIM, perante o aumento de casos críticos de COVID-19. Metodologia. Realizou-se um estudo descritivo com os dados dos tabuleiros de controle da Direção de Urgências e os indicadores de SaluData. Contrastaram-se os pontos essenciais da estratégia distrital e a porcentagem de ocupação nos serviços UCI e UCIM com corte a cada 12 horas. Resultados. Descreve-se o desenho e a implementação da estratégia; os indicadores de porcentagem de ocupação específicos para camas UCI, UCIM; os indicadores de hospitalização para casos de COVID-19 e não COVID-19; e os parâmetros de alta demanda em um rango maior ou igual a 50 em porcentagem de ocupação camas UCI adulto, por um período contínuo maior a 24 horas. Determinaram-se os níveis de alerta tendo em conta tais porcentagens. Igualmente, foram tomados em conta os procedimentos, instrumentos e canais de comunicação necessários para a regulação da oferta de camas. Conclusão. A gestão centralizada de camas UCI por parte do Centro Regulador tem favorecido a prestação de serviços de saúde a pacientes em estado crítico.

Palavras-chave: Infecções por Coronavírus, unidades de terapia intensiva, serviços de saúde, ocupação de leitos, conversão de leitos. 
Gestión centralizada de camas de Unidad de Cuidado Intensivo e Intermedio por

el Centro Regulador de Urgencias y Emergencias de Bogotá ante el COVID-19

\section{Introducción}

La COVID-19 también conocida como enfermedad por nuevo coronavirus es causada por el coronavirus 2 del síndrome respiratorio agudo severo (SARS-CoV-2) considerada como Pandemia por la Organización Mundial de la Salud (OMS), produce síntomas similares a los de la gripe, entre los que se incluyen fiebre, tos, disnea, mialgia y fatiga (1).

En noviembre de 2019 se produjo el primer caso confirmado de contagio de SARS\#CoV-2. La provincia China de Hubei fue inicialmente la más afectada, lo que llevó al gobierno a decretar una estricta cuarentena domiciliaria y cordones sanitarios para la contención del virus. Estas estrategias lograron en gran medida contener el brote. El primer caso fuera de China fue detectado el 13 de enero de 2020 en Tailandia, a partir de ese momento se comienzan a evidenciar fuertes diferencias en la incidencia de la pandemia a medida que el virus se propagaba en el mundo. Es así como la respuesta de los países comienza a adquirir distintos énfasis en función de las contingencias, enfoques y recursos locales. Posteriormente la pandemia se traslada a Europa, donde algunos países como Italia, España y Gran Bretaña son fuertemente afectados. En Italia se vivieron momentos complejos por la saturación del sistema de salud. Frente a este preocupante avance de los contagios y el incremento de las defunciones la mayoría de los países europeos adoptaron medidas estrictas de confinamiento, como se había hecho en la ciudad china de Wuhan.

Algunos países como Gran Bretaña, Holanda y Suecia ensayaron un modelo alternativo más flexible que buscaba estimular la adquisición de la inmunidad a nivel poblacional. Sin embargo, ante la letalidad que se comenzó a evidenciar, solo Suecia persistió en ese modelo, evidenciando a comienzos de junio de 2020 un descenso en su tasa de defunciones mucho más lento que los países que optaron por los confinamientos obligatorios. E1 31 de enero, diez días después de la llegada del virus a los Estados Unidos, cerró sus fronteras a los extranjeros buscando contener el avance del virus. Toda una ola de cierres de fronteras se produjo en corto tiempo que, sólo a fines de mayo, comenzó a ser reevaluada en algunas zonas específicas en las que se evidenció un cierto control de la epidemia como en Australia y Nueva Zelanda. Ya en suelo americano el virus no tardó en alcanzar Latinoamérica y el Caribe, confirmándose el primer caso en Brasil el 26 de febrero, el 28 del mismo mes en México, el $1^{\circ}$ de marzo en Ecuador y República Dominicana y el 6 de marzo en Colombia. A poco más de cuatro meses de declarada la pandemia a nivel mundial por la OMS, la región de las Américas se convirtió en el nuevo epicentro de contagios y defunciones (2).

Al 16 de noviembre de 2020 el SARS-CoV-2 había afectado a más de 55,2 millones de personas y había causado más de 1,33 millones de muertes en 216 países. La región de las Américas ha reportado alrededor de 23,4 millones de casos y 680.800 defunciones, es decir que esta región ha aportado el 51,1\% del total de las muertes en el mundo por COVID-19 (3). Según datos reportados por el Instituto Nacional de Salud, en Colombia ha afectado a cerca de 1,2 millones de personas y ha causado alrededor de 34.223 defunciones, con una tasa de letalidad de $2,8 \%$ y una positividad acumulada de $24,3 \%$. Entre los casos activos el 70,0\% están en casa, en hospitalización general se reportan el $26,2 \%$, en unidades de cuidados intensivos el 3,7\%. El 
$76,4 \%$ de los fallecidos están en el grupo de edad de 60 y más años. Las personas que presentan el mayor número de muertes están entre 70 a 79 años con 8.562, seguidas del grupo de 60 a 69 años con 7.545 fallecidos (4).

La información disponible indica que aproximadamente entre el $80 \%$ y el $85 \%$ de las personas con COVID-19 desarrollarán síntomas leves y no requerirán asistencia hospitalaria, mientras que un $15 \%$ precisará cuidados intermedios o en salas generales, y el $5 \%$ restante requerirá de asistencia en unidades de cuidados intensivos (5). Las fases de una pandemia las define la Organización Mundial de la Salud para cada condición en particular, para SARs-CoV-2 estableció cuatro fases: 1-preparación, 2-contención, 3-contagio comunitario (con mitigación) y 4-transmisión sostenida (6).

Durante la emergencia sanitaria la respuesta de los países de la región ha sido diversa, ante la inexistencia de una vacuna y por la incertidumbre con respecto al tiempo para adquirir la inmunidad poblacional. Las medidas no farmacéuticas o de comportamiento social han estado en la primera línea de acción frente a la pandemia y las medidas de mitigación y contención han ocupado un papel crucial para evitar la saturación hospitalaria y el colapso de los sistemas de salud (7).

Las medidas de contención buscan minimizar el riesgo de transmisión hacia las personas no infectadas y las medidas de mitigación buscan, una vez que el virus ya circula en una población, reducir el pico de demanda de servicios de salud (8). La contención es efectiva en las fases iniciales de un brote contagioso, en tanto las medidas de mitigación se deben aplicar cuando el brote ya está avanzado y muchas personas han contraído el virus. Entre las medidas de contención se puede mencionar el cierre de fronteras internacionales, los cordones sanitarios entre territorios de un mismo país, el cierre de colegios y universidades, la prohibición de reuniones y la promoción del teletrabajo. Por su parte las medidas de mitigación más relevantes son los confinamientos domiciliarios, las medidas de distanciamiento social en las interacciones cotidianas de las personas, el uso de dispositivos de protección, la expansión hospitalaria y la reorganización de los servicios de salud.

En paralelo a la contención y a la mitigación extrahospitalaria, las respuestas políticas a la pandemia se aplican en los servicios de salud donde se coordinan y se prestan los cuidados y tratamientos que pueden salvar las vidas de quienes han desarrollado cuadros severos de la enfermedad. El refuerzo y la reorganización de los servicios de salud ocupan el centro de esta política que, a través de distintas estrategias, buscan darles la atención más pronta y adecuada a los pacientes y evitar la sobrecarga del sistema en zonas geográficas o dispositivos específicos que puedan presentar alta demanda en corto tiempo (2).

Según la Organización para la Cooperación y el Desarrollo Económicos hay cuatro estrategias claves que los sistemas de salud están implementando para enfrentar la pandemia: el refuerzo 
y la optimización de la capacidad de respuesta ante el rápido aumento de la demanda, asegurar el acceso de los más vulnerables a los diagnósticos y tratamientos, las respuestas digitales y de datos para fortalecer tanto la vigilancia epidemiológica como los tratamientos y, finalmente, las mejoras en investigación y desarrollo I+D (9).

Un estudio realizado por el Comité de gestión, calidad y escores de la Sociedad Argentina de Terapia Intensiva, basado en la experiencia previa ante otras pandemias (SARS, MERS e Influenza H1N1) y en la experiencia actual internacional, proporciona recomendaciones y sugerencias para la organización, preparación y gestión de las Unidades de Cuidado Intensivo (UCI) que aseguren la continuidad de la atención clínica teniendo en cuenta las diferentes fases de una pandemia por COVID-19. Enuncian que, durante una pandemia se pueden presentar estas etapas: preparación (pre-pandémica), etapa 1 inicio de la pandemia, etapa 2 Saturación de la UCI, etapa 3 Colapso de la UCI y de la institución y etapa 4 Control de la crisis (10).

Dentro de las recomendaciones para planificar la respuesta ante una pandemia el recurso más importante en las UCI es el personal experimentado en cuidados intensivos. Se recomienda un enfoque sistémico que involucre todas las organizaciones, más un trabajo conjunto entre hospitales privados y públicos, UCI de adultos y pediátricas y, si estuvieran disponibles, favorecer acuerdos de Telesalud para apoyar a las UCI de diferentes niveles para asegurar la prestación justa y equitativa de atención a todos los pacientes. A nivel institucional es muy importante que cada centro desarrolle sus planes para enfrentar la pandemia y prepare a las UCI propias, alineándolas con las políticas regionales. Estos planes deben adoptar una respuesta gradual y escalonada basada en el impacto de la pandemia sobre la capacidad de la UCI para satisfacer las necesidades operativas diarias e incluir enfoques operativos para reducir la demanda rutinaria de la UCI, identificar y aumentar la capacidad física de camas de la UCI en todo el hospital, determinar las necesidades conexas de recursos humanos y equipamiento y evitar el agotamiento del recurso humano, así como proteger su seguridad (10).

La Sociedad de terapia intensiva proporciona algunas recomendaciones para implementar en las diferentes fases o etapas de una pandemia. Para la fase 1: revisar y poner a prueba los planes de respuesta a la pandemia incluyendo infraestructura y equipo, capacitación, planificación y apoyo de la fuerza de trabajo, los planes de comunicación, el control de las infecciones, los protocolos de diagnóstico y tratamiento, las políticas de transporte y transferencia, el aseguramiento anticipado de puntos de activación para pasar a una respuesta de mayor nivel. Para la fase 2: establecer medidas para reducir la demanda y aumentar la capacidad física, designar áreas clínicas alternativas para el cuidado de pacientes con necesidad de cuidados críticos no ventilados, considerar las necesidades de fuerza de trabajo y de personal y diferir o derivar cirugía no urgente a otros centros u otros servicios. Para la etapa 3: reaprovechar las áreas clínicas alternativas para pacientes ventilados, considerar la posibilidad de trasladar a los pacientes a otras instalaciones o identificar recursos adicionales para ser transferidos al hospital para facilitar la atención continua de la UCI, reevaluar los criterios de admisión y egreso de UCI y considerar brindar cuidados críticos en zonas no convencionales. Finalmente para la etapa 4: 
reorganizar las actividades en la institución, evaluar la respuesta brindada durante la crisis y proponer mejoras a implementar en los planes futuros (10).

A la fecha de corte de los datos de esta investigación, 16 de noviembre de 2020, Colombia se encuentra en la tercera fase de la pandemia correspondiente a la mitigación, en la cual se busca la reducción del impacto epidémico y de la tensión en el sistema de salud, mediante el retraso del pico del brote, la disminución del número máximo de casos, la implementación de estrategias más exigentes de búsqueda activa de casos en la población general por parte del sector salud y la compensación de los efectos de la crisis económica y del mercado laboral por parte de los demás sectores.

La primera fase de preparación, vigilancia y prevención culminó el 6 de marzo de 2020 con la confirmación del primer caso de COVID-19 en Colombia; posteriormente cursó la segunda fase de contención, procurando disminuir la frecuencia de contagios (11). Durante el desarrollo de esta fase, los gobiernos nacional y distrital adoptaron medidas relacionadas con aislamientos obligatorios, preventivos y voluntarios, medidas de autocuidado y distanciamiento físico, entre otras. De la misma forma expidieron normativas encaminadas a paliar la crisis y a impedir la extensión de sus efectos.

Después de las recomendaciones dadas por la Organización Panamericana de la Salud (OPS), la experiencia de lo ocurrido en países europeos y el pronóstico realizado a través de la modelación matemática que evidenciaba un incremento progresivo en el requerimiento de la capacidad instalada hospitalaria a nivel nacional; el Ministerio de Salud y Protección Social expidió el Decreto 538 del 12 de abril de 2020 en el que se adoptan medidas en el sector salud para contener y mitigar la pandemia de COVID-19 y garantizar la prestación de los servicios de salud. Una de estas medidas corresponde a la de facultar a las entidades territoriales para que en caso de alta demanda, asuman el control de la oferta y disponibilidad de las UCI y de las unidades de cuidados intermedios (UCIM) que están bajo el control de las Empresas Administradoras de Planes de Beneficios (EAPB) por medio de los Centros Reguladores de Urgencias, Emergencias y Desastres (CRUE) (12).

Por las deficiencias evidenciadas en el sistema de salud colombiano desde la promulgación de la Ley 100 en 1993 es difícil que se garantice que toda persona enferma acceda oportunamente a un servicio de salud con calidad aun teniendo cobertura universal (13). Para algunos, el Sistema General de Seguridad Social en Salud (SGSSS) ha orientado la salud hacia actividades comerciales de mala calidad y excluyentes, y las Entidades Promotoras de Salud (EPS) ponen trabas al goce efectivo del derecho por parte de sus usuarios, obligándolos a acudir a la vía judicial para garantizar los derechos fundamentales mediante la acción de tutela como mecanismo de amparo existente en el país (14). 
Para la OPS el objetivo principal de los servicios de salud en respuesta al COVID-19 es salvar vidas, más aun observando que en la letalidad del cuadro clínico influye en forma decisiva la capacidad de respuesta de la red asistencial, especialmente la identificación temprana de casos y la disponibilidad y acceso a cuidados críticos proporcionados por personal de salud suficiente y competente. También insta a los países miembros a adoptar medidas para avanzar en la reorganización y ampliación progresiva de la capacidad de respuesta de los servicios de salud, tales como la gestión centralizada de camas y la coordinación con la red de servicios de atención prehospitalaria $(15,16)$.

Bogotá siendo un entorno urbano con complejos asentamientos poblacionales, diversas vulnerabilidades sanitarias y desigualdades sociales (17), inicia la adaptación de las directrices impartidas por el Gobierno Nacional en cuanto a la generación de lineamientos que buscan articular a los integrantes del SGSSS, bajo los principios de subsidiariedad, complementariedad, concurrencia y unidad con el fin de acoger medidas en procura de evitar, contener o mitigar los posibles efectos que ocasione esta situación epidemiológica en el Distrito Capital (18).

Por otra parte, es preciso mencionar que en la actual alerta sanitaria se pueden entrever consecuencias relacionadas con el incremento de las brechas sociales y económicas en la población mundial, uno de los aspectos que generaría esta disparidad tiene que ver con las condiciones de salud y el acceso a sus servicios. El acceso a los servicios sanitarios es más difícil para las personas con menos recursos que para los privilegiados económicamente $(19,20)$. Los resultados de un estudio desarrollado en Barcelona sobre la incidencia acumulada de COVID-19 confirmada por PCR, sugiere que los determinantes sociales de la salud se correlacionan con una modificación de la incidencia de la COVID-19 en los barrios de la ciudad, existiendo un mayor riesgo de contraer la enfermedad en aquellos más limitados económicamente (21).

Este artículo busca presentar la estrategia implementada en Bogotá para la coordinación de camas de Unidad de Cuidado Intensivo e Intermedio (UCI) establecida desde la Dirección de Urgencias y Emergencias en Salud de la Secretaria Distrital de Salud de Bogotá frente el aumento de casos críticos de COVID-19 en el marco de la emergencia sanitaria, así como los resultados de su operación.

\section{Métodos}

Se realizó un estudio descriptivo para presentar la estrategia de coordinación de las camas de cuidados intensivos y cuidados intermedios, implementada en el Distrito Capital durante el año 2020 como medida decretada por el gobierno de Colombia en el marco de la emergencia sanitaria por COVID-19, junto con los resultados obtenidos después de su implementación.

Para la descripción de la estrategia se tomó la información registrada en el Decreto 538 de 2020 expedido por el Ministerio de Salud y Protección Social y en el Lineamiento técnico base para la operación del manejo integral de las unidades de cuidado intensivo e intermedio ante la emergencia COVID-19. Para la evaluación de los resultados de la implementación y operación 
se tomaron los datos registrados en los tableros de control y seguimiento de la Dirección de Urgencias y Emergencias en Salud, junto con los datos de los porcentajes de ocupación de las UCI y UCIM publicados por el Observatorio de Salud SaluData de la Secretaria Distrital de Salud.

Para la recolección de los datos de la operación de la estrategia se tuvieron en cuenta las variables categóricas: tipo de Servicio (UCI, UCIM, Hospitalización), tipo de servicio de acuerdo con el grupo de pacientes atendidos (adulto, pediátrico, neonatal), tipo de institución destino del traslado (pública o privada), desenlace de la solicitud (autorizada, fallida), condición final del paciente antes del traslado (vivo, muerto), tipo de Instituciones Prestadoras de Servicios (IPS) autorizada para el traslado (igual o diferente a la solicitada) y tipo de servicio de acuerdo a la patología (COVID - no COVID) y para el análisis de la información se planteó el indicador porcentaje de ocupación en cada uno de los servicios realizando dos mediciones diarias con corte cada 12 horas.

\section{Resultados}

\section{Diseño e implementación de la estrategia}

La generación de la alerta de alta demanda en el sector salud de Bogotá D.C. se estableció una vez se cumplieron dos aspectos: el primero, que el indicador de ocupación de camas de UCI general adulto se encontrara en un rango mayor o igual al 50\% según lo publicado por la Secretaría Distrital de Salud en el Observatorio de Salud de Bogotá SaluData; y el segundo, que esta situación se observara por un periodo continuo de tiempo mayor a 24 horas. Asimismo, se establecieron los niveles de alerta en la ciudad de Bogotá frente al resultado de la medición del indicador de Porcentaje de Ocupación de UCI COVID-19, teniendo como base lo establecido por el Ministerio de Salud y Protección Social en el documento Guía Hospitalaria para la Gestión del Riesgo de Desastres y en el sistema de alertas o escalas de alertas en el sector salud (verde, amarilla, naranja y roja). Este último provee medidas de pronóstico y preparación, relacionadas con dos aspectos: la información previa que existe sobre la evolución de un fenómeno y las acciones y disposiciones que deben ser asumidas por los Comités para la Prevención y Atención de Desastres para enfrentar la situación que se prevé (13).

Para hacer el seguimiento a la ocupación de las camas se establecieron indicadores específicos que corresponden al porcentaje de ocupación para los servicios de unidad de cuidados intensivos, unidad de cuidados intermedios y hospitalización de adultos, pediátricos y neonatos de los casos probables o confirmados para COVID-19 y los no COVID-19, atendidos en las IPS públicas y privadas que tienen habilitados estos servicios (13), el seguimiento se realizó dos veces al día con corte cada 12 horas. 
Cabe resaltar que desde el mes de junio de 2020 hasta el 28 de septiembre de 2020 los niveles de alerta se establecían con la medición del porcentaje de ocupación de las camas de UCI destinadas exclusivamente para la atención de pacientes COVID-19, y a partir del 29 de septiembre de 2020 se amplió el cálculo del indicador incluyendo en el denominador la totalidad de las camas UCI disponibles, dicha ampliación obedeció a la disminución de los casos COVID-19 en UCI y la evidente necesidad de disponer de la capacidad instalada para atender los pacientes con requerimientos de camas de UCI por otro tipo de patologías. Los niveles de alerta se establecieron de acuerdo con lo establecido en la Resolución 1698 de 2020. Ver tabla 1.

Tabla 1 Niveles de alerta de Bogotá de acuerdo con la Resolución 1698 de 2020

\begin{tabular}{|l|l|l}
\hline \multicolumn{1}{|c|}{$\begin{array}{c}\text { Rango } \\
\text { (Porcentaje de ocupación UCI COVID-19) }\end{array}$} & Tipo de alerta & Nivel de riesgo \\
\hline Entre 0 y $29 \%$ & Verde & Bajo \\
\hline Entre 30 y $49 \%$ & Amarilla & Moderado \\
\hline Entre 50 y $74 \%$ & Naranja & Alto \\
\hline Igual o mayor al $75 \%$ & Roja & Muy alto \\
\hline
\end{tabular}

Fuente: Secretaría Distrital de Salud. Resolución 1698 del 9 de septiembre de 2020.

Para regular la oferta de camas de UCI y UCIM una vez establecida la alta demanda se procedió a organizar la respuesta a través del CRUE, se incrementó el número de médicos reguladores, enfermeros, técnicos y gestores de información; se acondicionó un área exclusiva para la regulación de las camas de UCI; se pusieron en funcionamiento los tableros de control diseñados por la Dirección de Tecnologías de información para conocer en tiempo real la disponibilidad y ocupación de las camas hospitalarias; se pusieron en funcionamiento los módulos del aplicativo SIDCRUE para recibir las solicitudes de camas de UCI, registrar las decisiones, las actividades y almacenar los soportes, regular el traslado secundario de los pacientes con los vehículos de emergencia tipo ambulancias medicalizadas dispuestos por las EAPB y por la Secretaría Distrital de Salud y emitir los Códigos QR respectivos para las IPS receptoras de los pacientes. Y se reorganizó el equipo con el fin de cumplir de manera ágil con los traslados requeridos de acuerdo con la disponibilidad de camas, así como minimizar los trámites administrativos existentes en los procesos de referencia y contrarreferencia (13).

Mientras se presentó la alta demanda en los servicios de salud, la Secretaria Distrital de Salud estableció medidas de obligatorio cumplimiento por parte de las IPS y las EAPB en relación con el talento humano, los equipos biomédicos, las actividades ambulatorias, los procedimientos quirúrgicos ambulatorios y programados y el manejo de las camas hospitalarias y de UCI e UCIM, con un seguimiento estricto a los prestadores de la ciudad con énfasis en los ubicados en las zonas de cuidado especial.

Una de las medidas establecidas fue el reporte de las proyecciones de ampliación o reconversión de camas hacia UCIM e UCI para adulto, tanto para pacientes COVID como no COVID, así como la disponibilidad de equipos biomédicos para este fin. Además, cada IPS debía dar continuidad al reporte del Sistema Integral de Referencia y Contrarreferencia (SIRC) para todos los servicios de hospitalización general, unidad de cuidados intermedios e intensivos tanto 
para pacientes COVID como no COVID e inscribir en el módulo de autorización de servicios transitorios del Registro Especial de Prestadores el servicio de unidad de cuidado intensivo adulto y su capacidad instalada, para obtener la correspondiente autorización en los términos definidos en el artículo 1 del Decreto 538 del 12 de abril de 2020.

En la estrategia de coordinación se estableció que una vez la IPS identificara en su servicio de urgencias a un paciente que requiriera el traslado a una unidad de cuidados intermedios o intensivos debía comunicarse de forma inmediata con el CRUE de la Secretaría Distrital con el fin de documentar los datos y el estado del paciente, para que el CRUE direccionara el paciente a la IPS seleccionada según criterio territorial o con mayor disponibilidad del servicio requerido, se comunicara con la IPS receptora del paciente, activara la empresa de ambulancia medicalizada contratada por la EAPB responsable del paciente para realizar el traslado secundario y le emitiera el código tipo QR respectivo a la IPS una vez fuera recibido el paciente. Por otro parte, la IPS receptora del paciente debe registrar en el aplicativo SIRC del Sistema Integral de Referencia y Contrarreferencia la novedad de ingreso a los servicios de forma inmediata, al igual que la de egreso en el momento que se suceda el hecho.

Finalmente, se estableció que sobre los médicos reguladores no debía recaer la toma de decisión sobre los dilemas que se presentan en la atención de pacientes críticos afectados por COVID-19, en contextos de escasez, limitación de recursos y de alta demanda de insumos y atención médica (4), dicha decisión es responsabilidad de los médicos tratantes de las instituciones remitente y receptora del paciente, con esto se busca que para todos los pacientes potencialmente afectados, se aseguren los más altos estándares éticos y clínicos en la toma de decisiones $(17,18)$.

\section{Regulación de UCI y UCIM por parte del CRUE}

A partir del 16 de junio de 2020 el CRUE de la Dirección de Urgencias y Emergencias en Salud asumió la regulación de las camas de UCI COVID cuyo porcentaje de ocupación se encontraba en el 56,9\%, con 408 de 734 camas ocupadas. Del 16 de junio al 16 de noviembre de 2020, el CRUE del Distrito recibió 11.487 solicitudes para la ubicación de pacientes complicados con síntomas de infección por COVID-19 en una unidad de cuidado intensivo o intermedio, de estas el $85,8 \%(\mathrm{~N}=9.857)$ fueron autorizadas, el $10,2 \%(\mathrm{~N}=1.175)$ fueron canceladas por parte de la IPS que realizó la solicitud y 4,0\% (N=455) se anuló por fallecimiento del paciente en la IPS remisora.

De las 9.857 solicitudes de camas de unidad de cuidado intensivo e intermedio para COVID-19 autorizadas, el 76,7\% (N=7.556) correspondieron a UCI adultos, el 18,8\% (N=1.858) a UCIM adultos, el $1,8 \%(\mathrm{~N}=173)$ a UCI pediátricas, el $0,5 \%(\mathrm{~N}=51)$ a UCIM pediátricas, el $1,7 \%$ $(\mathrm{N}=166)$ a UCI neonatales y el $0,5 \%(\mathrm{~N}=53)$ a UCIM neonatales. 
El pico máximo de solicitudes se registró entre el 24 y el 27 de julio, recibiendo para el día 26 de

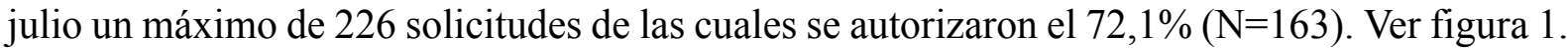

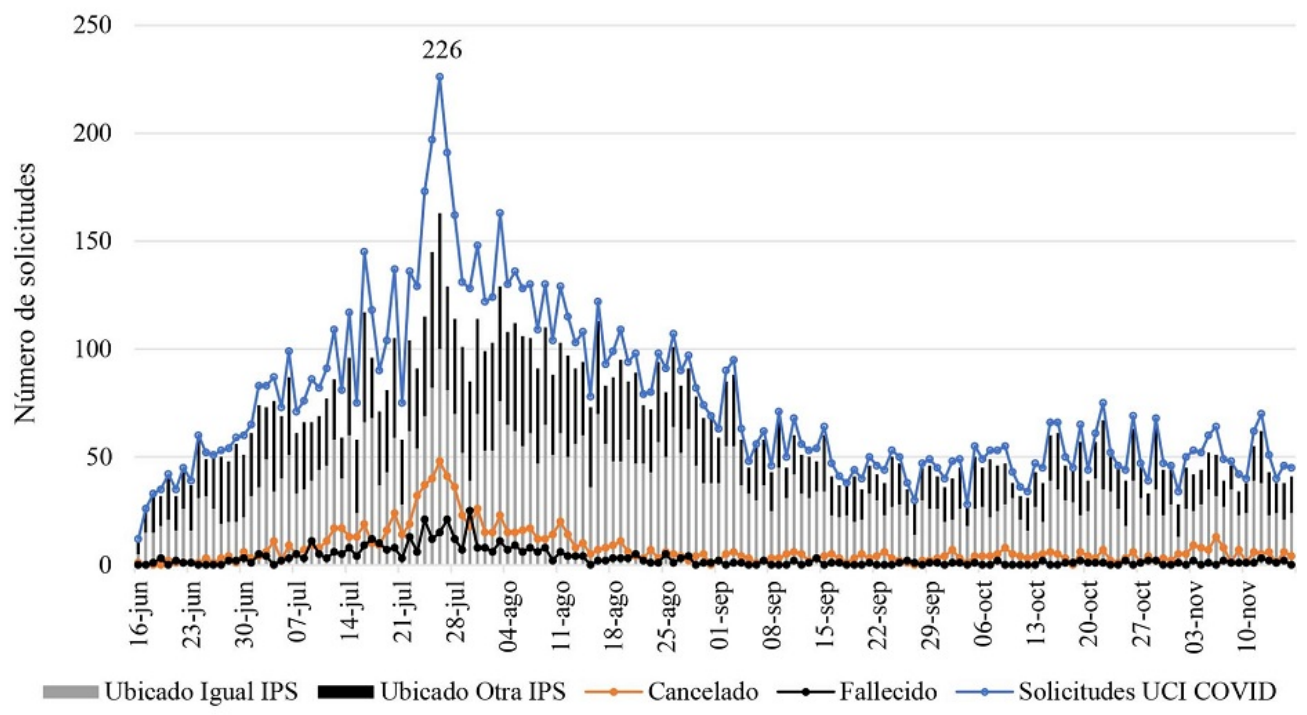

Figura 1 Solicitudes de UCI adulto COVID

gestionadas desde el CRUE, Bogotá, junio-nov., 2020

Fuente: Dirección de Urgencias y Emergencias en Salud, tableros de control y seguimiento \% de ocupación UCI y UCIM COVID, no COVID. Bogotá, 16 de junio - 16 de noviembre de 2020.

Por otro lado, del 23 de julio al 23 de noviembre de 2020 se recibieron 8.698 solicitudes para la ubicación de pacientes complicados no COVID en unidades de cuidado intensivo o intermedio, de estas el 88,6\% $(\mathrm{N}=7.705)$ fueron autorizadas, el 9,5\% $(\mathrm{N}=828)$ fueron canceladas por parte de la institución prestadora del servicio que realizó la solicitud, el 1,5\% ( $\mathrm{N}=134)$ se anuló por fallecimiento del paciente en la IPS remisora y el 0,4\% (N=31) habían sido comentados en alguna UCI y estaban a la espera de autorización, cabe aclarar que estos últimos fueron comentados durante los últimos dos días del periodo analizado.

De las 7.705 unidades de cuidado intensivo e intermedio no COVID autorizadas, el $62,1 \%$ $(\mathrm{N}=4.784)$ correspondieron a UCI adultos, el $18,8 \%(\mathrm{~N}=1.447)$ a UCIM adultos, el $4,7 \%$ $(\mathrm{N}=364)$ a UCI pediátricas, el $1,7 \%(\mathrm{~N}=131)$ a UCIM pediátricas, el $9,5 \%(\mathrm{~N}=730)$ a UCI neonatales y finalmente el $3,2 \%(\mathrm{~N}=249)$ a UCIM neonatales.

Las solicitudes más altas se registraron los días 10 de septiembre con 105 solicitudes, el 15 de octubre con 103 y el 8 de octubre con 100 solicitudes. Ver figura 2. 
Andrés José Álvarez Villegas / Ángela María Roa Saldaña / Edizabeth Ramírez Rodríguez

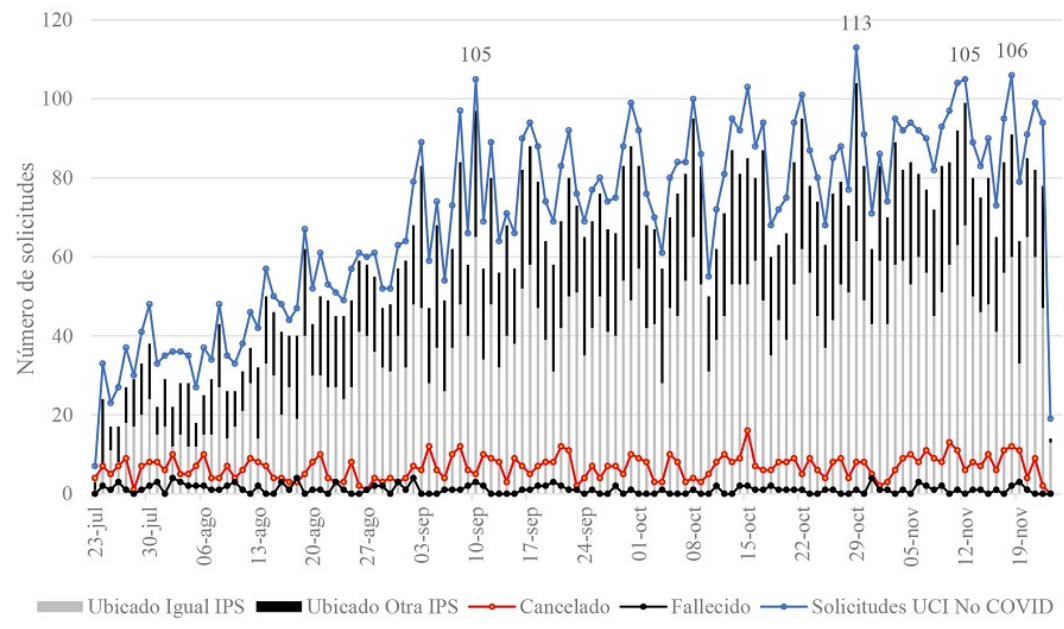

Figura 2 Solicitudes de UCI adulto no COVID gestionadas por el CRUE, Bogotá, julio-nov., 2020

Fuente: Dirección de Urgencias y Emergencias en Salud, tableros de control y seguimiento \% de ocupación UCI y UCIM COVID, no COVID. Bogotá, 23 julio a 23 de noviembre de 2020.

El número máximo de camas UCI asignadas para atención de COVID-19 en la ciudad se alcanzó el 7 de septiembre con 1.882 camas, el 53,5\% de estas se ubicó en las localidades de Usaquén, Chapinero, Teusaquillo y Los Mártires. La Unidad de Planeamiento Zonal (UPZ) con mayor oferta correspondió a La Sabana, de la localidad Los Mártires, con 199 camas (10,6\%), seguido de la UPZ La Uribe en Usaquén con 159 camas $(8,4)$. Ver figura 3.

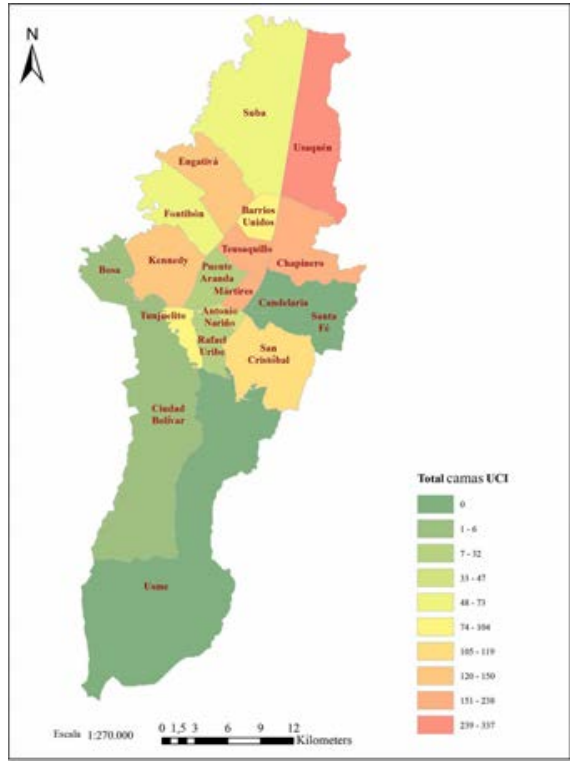

Figura 3 Número de camas UCI asignadas para atención de COVID-19 por localidad, Bogotá, 2020

Fuente: Dirección de Urgencias y Emergencias en Salud, tableros de control y seguimiento \% de ocupación UCI y UCIM COVID, no COVID. Bogotá, junio a octubre de 2020. 
El porcentaje de ocupación de camas UCI adulto COVID se demoró 16 días hasta alcanzar el límite inferior de la alerta roja, aumentando en un $20,5 \%$ al 2 de julio. Al final, su pico máximo fue el 26 de julio, llegando a un $93,2 \%$. A pesar del incremento de camas, la alerta roja se mantuvo hasta el 25 de septiembre de 2020 al alcanzar el 50,1\% de ocupación, a partir de este momento el indicador ha mantenido un comportamiento estable entre el rango entre $46,0 \%$ y $51,2 \%$. Ver figura 4 .

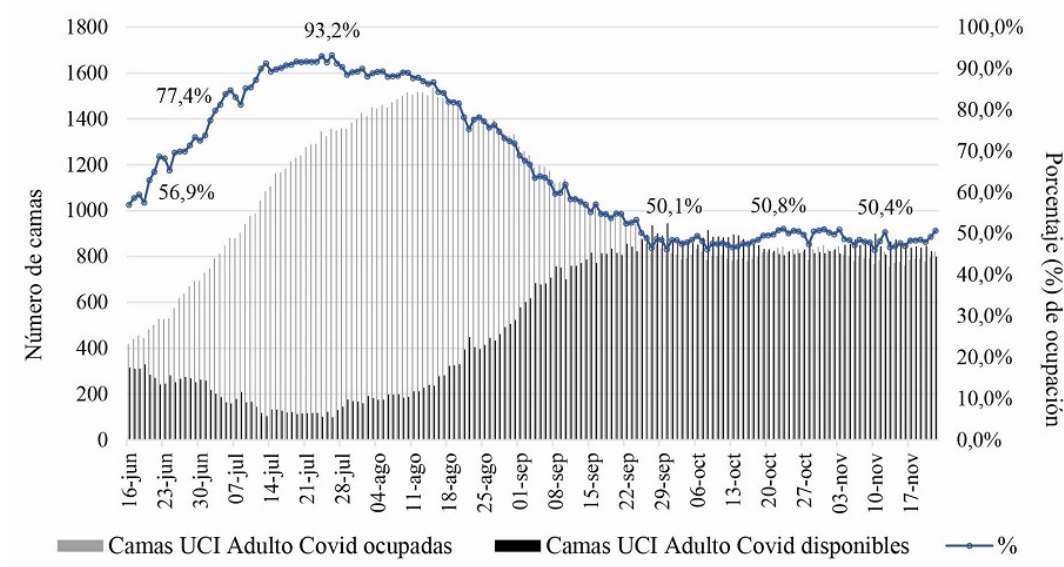

Figura 4 Ocupación de camas UCI adulto COVID, Bogotá, 16 de junio-16 de nov., 2020

Fuente: Dirección de Urgencias y Emergencias en Salud, Tableros de control y seguimiento \% de ocupación UCI y UCIM COVID, no COVID. Bogotá, 16 de junio a 16 de noviembre de 2020.

El 26 de julio de 2020 para las IPS (N=57) que tenían asignación de camas UCI para atención de pacientes COVID-19, el 60\% presentaba un porcentaje de ocupación del 100\%, y se ubicaban principalmente en las localidades de Chapinero, Teusaquillo y Kennedy. Ver figura 5.

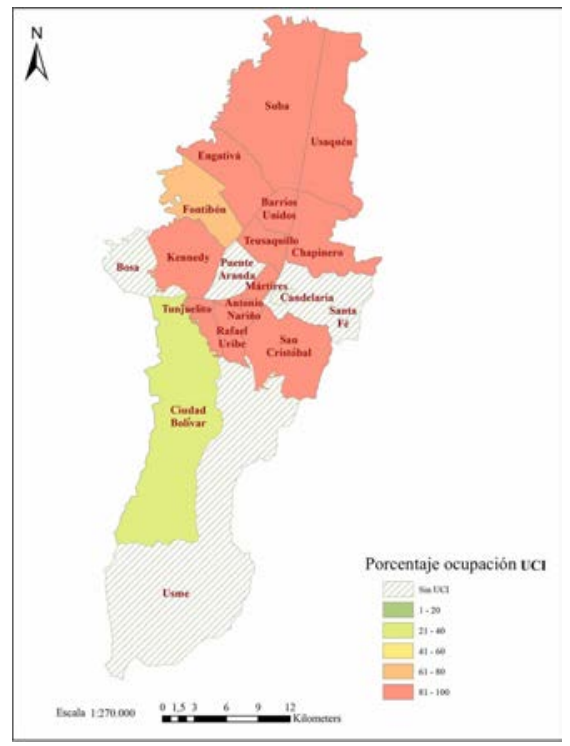

Figura 5 Porcentaje de ocupación de camas UCI asignadas para atención de COVID-19 por Localidad, Bogotá, 2020

Fuente: Dirección de Urgencias y Emergencias en Salud, Tableros de control y seguimiento porcentaje de ocupación UCI y UCIM COVID, no COVID. Bogotá, junio a octubre de 2020. 


\section{Conclusiones}

La gestión centralizada de las camas de UCI favorece la atención oportuna de los pacientes con enfermedad grave por COVID-19 y los pacientes con patologías no COVID-19, minimizando las barreras administrativas de acceso a estos servicios de salud especializados.

Las decisiones clínicas del manejo de los pacientes son tomadas por los médicos tratantes, tanto en las instituciones remisoras como en las receptoras de acuerdo con los protocolos establecidos por cada una de ellas, debido a que los médicos reguladores no son los médicos tratantes y actúan como personal administrativo que verifica que los registros, historia clínica y evoluciones estén completos, sean conocidos y aprobados por los intensivistas de las IPS receptoras, es decir, que son esenciales en la gestión efectiva de las camas de cuidado intensivo e intermedio disponibles en la red hospitalaria.

La pandemia está agravando la carga de morbilidad y el acceso oportuno a los servicios de salud, con mayor relevancia en las poblaciones con más desigualdades sociales, por lo que la gestión centralizada de las camas de cuidado intensivo podría considerarse como una estrategia que cubra el periodo posterior a la pandemia.

Como limitación del estudio se encuentra la evaluación de indicadores de mejoramiento en el acceso a servicios de cuidado intensivo demandados por pacientes COVID y no COVID.

\section{Consideraciones éticas}

De acuerdo con lo establecido en la Resolución 8430 de 1993 expedida por el Ministerio de Salud la presente investigación no presentó ningún tipo de riesgo. La información recopilada se obtuvo a través de SaluData y los tableros de control de la Dirección de urgencias y emergencias en salud. La investigación se realizó con datos anónimos, los cuales no contenían información personal o identificable de los individuos en estudio.

\section{Contribución de los autores}

Los tres autores participaron según sus competencias de manera equitativa en la construcción, revisión y ajustes del artículo.

\section{Financiación}

Este estudio fue realizado con recursos propios de la institución.

\section{Conflicto de intereses}

Los autores no han referido ningún potencial conflicto de interés. 
Gestión centralizada de camas de Unidad de Cuidado Intensivo e Intermedio por

el Centro Regulador de Urgencias y Emergencias de Bogotá ante el COVID-19

\section{Agradecimientos}

A la Dirección de urgencias y emergencias en salud y la Subdirección Centro regulador de urgencias y emergencias de la Secretaría Distrital de Salud de Bogotá por el suministro de información y por las aclaraciones pertinentes.

\section{Referencias}

1. Perez M, Gomez J, Dieguez R. Características clínico-epidemiológicas de la COVID-19. Rev Habanera Cienc Médi. 2020;19(2). https://www.medigraphic.com/cgi-bin/new/resumen.cgi?IDARTICULO=9 7798

2. Moller S. Intervenciones sociosanitarias y uso de las tecnologias de la industria 4.0 para enfrentar la enfermedad por coronavirus (COVID-19) en America Latina y el Caribe. Politicas sociales. Naciones Unidas, Santiago: CEPAL; 2020.

3. Organización Panamericana de la Salud - Organización Mundial de la Salud. Reporte situación COVID - 19 Colombia; 2020. https://www.paho.org/es/documentos/reporte-situacion-covid-19-colombia-no -177-11-noviembre-2020.

4. Maglio I, Valdes P, Camera L, Finn B, Klein M, Pincemin I, et al. Guías éticas para la atención durante la pandemia COVID-19. Medicina Buenos Aires. 2020;80(3). https://www.medicinabuenosaires.co $\mathrm{m} /$ revistas/vol80-20/s3/45.pdf

5. Asociación colombiana de infectología - Instituto de Evaluación Tecnológica en Salud. Consenso Colombiano de atención, diagnóstico y manejo de la infección por SARS-CoV-2/COVID 19 en establecimientos de la atención de la salud. Rev Asociación Colombiana Infectología. 2020;24(3). h ttp://dx.doi.org/10.22354/in.v24i3.851

6. Ministerio de Salud y Protección Social - República de Colombia. Canasta de tecnologías en salud y servicios para la atención de pacientes con COVID -19; 2020. https://www.minsalud.gov.co/sites/ri d/Lists/BibliotecaDigital/RIDE/VP/DOA/metodologia-resultados-canastas.pdf.

7. Ministerio de Salud y Protección Social. Decreto Legislativo No. 538 de 2020 Por el cual se adoptan medidas en el sector salud, para contener y mitigar la pandemia de COVID-19 y garantizar la prestación de los servicios de salud.

8. Bernal O. ¿Qué tipo de cambio necesita el sistema de salud en Colombia? Revista de asuntos políticos. 2011;8(Oct.):41-43.

9. Mendieta D, Jaramillo CH. El sistema general de seguridad social en salud de Colombia. Universal, pero ineficiente: a propósito de los 25 años de su creación. Rev Latinoam Derecho Social. 2020;(29). ht tps://doi.org/10.22201/iij.24487899e.2019.29.13905

10. Organización Panamericana de la Salud - OPS. Reorganización y ampliación progresiva de los servicios de salud para la respuesta a la pandemia de COVID-19. 2020. https://iris.paho.org/bitstream/handle/ 10665.2/52214/OPSHSSHSCOVID-19200018_spa.pdf? sequence=1\&isAllowed=y.

11. Mendoza C, Suárez M. Reconversión hospitalaria ante la pandemia de COVID-19. Rev Mex Anestesiol. 2020;43(2):10.35366/92875. https://dx.doi.org/10.35366/92875 
12. Organización Mundial de la Salud. Fortalecimiento de la preparación para la COVID-19 en las ciudades y otros entornos urbanos; 2020. https://apps.who.int/iris/bitstream/handle/10665/332039/WHO-201 9-nCoV-Urban_preparedness-2020.1-spa.pdf?sequence=1\&isAllowed=y.

13. Secretaria Distrital de Salud. Alcaldía Mayor de Bogotá DC. Lineamiento técnico base para la operación del manejo integral de las unidades de cuidado intensivo e intermedio ante la emergencia COVID-19 en el marco del Decreto 538 de 2020. http://www.saludcapital.gov.co/Documents/Covid_S/Lineam _camas_UCI_Dcto_538.pdf.

14. Rodríguez-Bailón R. Inequality viewed through the mirror of COVID-19 (La desigualdad ante el espejo del COVID-19). Int J Social Psychol. 2020;35(3):590-599. https://doi.org/10.1080/02134748.2020. 1796298

15. Organización Mundial de la Salud y PNUD. Hacer frente a las enfermedades no transmisibles durante la pandemia de COVID-19 y después de ella; 2020. https://apps.who.int/iris/bitstream/handle/10665 /335827/WHO-2019-nCoV-Non-communicable_diseases-Policy_brief-2020.1-spa.pdf.

16. Amengual M, Calafat M, Carot A, Rosa AR, Rio C, Rovira J, et al. Determinantes Sociales de la Incidencia de la COVID-19 en Barcelona: un estudio ecológico preliminar usando datos públicos. Rev Esp Salud Pública. 2020(Sept.);94. https://www.mscbs.gob.es/biblioPublic/publicaciones/recur sos_propios/resp/revista_cdrom/VOL94/ORIGINALES/RS94C_202009101.pdf

17. Rueda E, Caballero A, Bernal D, Torregosa L, Suárez E. Pautas éticas para la asignación de recursos sanitarios escasos en el marco de la pandemia por COVID-19 en Colombia. Rev Colomb Cir. 2020(abril);35. https://doi.org/10.30944/20117582.653

18. Asociación Colombiana de Medicina Crítica y Cuidado Intensivo. Declaración de consenso en medicina crítica para la atención multidisciplinaria del paciente con sospecha o confirmación diagnóstico COVID-19: Cap. 3. 2020. http://www.amci.org.co/images/consenso/Capitulo_3_Ingreso_del_pacie nte_a_UCI_Proce_dimiento_de_ingreso_y_traslado_hospitalario.pdf.

19. Secretaria Distrital de Salud de Bogotá. Alcaldía Mayor de Bogotá DC. Resolución 1698 del 09 de septiembre de 2020. 2020. Por la cual se declara la alerta naranja en el sistema hospitalario de Bogotá y se adoptan otras medidas.

20. Asociación Colombiana de Medicina Crítica y Cuidado Intensivo. Declaración de Consenso en Medicina Crítica para la Atención Multidisciplinaria del Paciente con Sospecha o Confirmación Diagnóstica COVID-19: Cap. 1. 2020 http://www.amci.org.co/images/consenso/Capitulo_1_Modelo_de_atencio n_y_escenarios_prioritarios_y_recursos.pdf.

21. Alvarez RP, Harris P. COVID-19 en América Latina: Retos y Oportunidades. Rev Chil Pediatr. 2020;91(2). http://dx.doi.org/10.32641/rchped.vi91i2.2157

22. Gozzer E, Canchihuamán F, Espinoza R. COVID-19 y la necesidad de actuar para mejorar las capacidades del Perú frente a pandemias. Rev Peru Med Exp Salud Pública. 2020;37(2). http://dx.d oi.org/10.17843/rpmesp.2020.372.5410

23. Ministerio de Salud y Protección Social. Plan de Acción para la prestación de servicios de salud durante las etapas de contención y mitigación de la pandemia por SARS-CoV-2 (COVID-19). $2020 \mathrm{https}: / / \mathrm{w}$ ww.minsalud.gov.co/Ministerio/Institucional/Procesos\%20y\%20procedimientos/PSSS01.pdf. 